\section{Strong Law of Large Numbers for Fuzzy Random Variables in Fuzzy Metric Space}

Reza Ghasemi, Mohammad Reza Rabiei, and Ahmad Nezakati

Faculty of Mathematical Sciences, Shahrood University of Technology, Shahrood, Iran
Received: Oct. 21, 2019

Revised : Feb. 26, 2020

Accepted: Mar. 17, 2020

Correspondence to: Mohammad Reza Rabiei (rabiei1354@yahoo.com)

(The Korean Institute of Intelligent Systems

(c) This is an Open Access article distributed under the terms of the Creative Commons Attribution Non-Commercial License (http://creativecommons.org/licenses/ by-nc/3.0// which permits unrestricted noncommercial use, distribution, and reproduction in any medium, provided the original work is properly cited.

\begin{abstract}
Despite uncertainty in fuzzy random variables, crisp metrics have always been used. In this study, we attempt to introduce the concept of fuzzy metric space and fuzzy normed space for fuzzy sets and some of its properties, and we investigate the strong law of large numbers. The embedded theorem for fuzzy compact sets in fuzzy normed space and the generalized Hukuhara difference are the most important tools used to prove this theorem. In addition, as a result and application, we use the strong law of large numbers for fuzzy random variables in the fuzzy metric space for the bootstrap mean.
\end{abstract}

Keywords: Fuzzy metric space, Limit theorems, Random set, Fuzzy random variable, Bootstrap mean

\section{Introduction}

Puri and Ralescu [1] provided a definition of a fuzzy random variable based on random sets. Because the spaces of fuzzy sets are not Banach spaces and they are not even vector spaces, Klement et al. [2] established the strong law of large numbers (SLLN) for the fuzzy random variables by embedding theorems. Lopez-Diaz [3] used a new approach for expressing and proving the SLLN by introducing simple convex random sets. In this approach, simple random sets were used. Colubi et al. [4] derived the SLLN for independent identically distributed (i.i.d) fuzzy random sets by using the approximation method of Lopez-Diaz and Gil [3]. In addition, Molchanov [5] demonstrated the SLLN for upper semicontinuous functions using a simpler approach. Li and Ogura [6] presented the SLLN for independent (not necessarily identically distributed) fuzzy-set-valued random variables in a separable Banach space or a Euclidean space. Kim [7] established some results on weak laws of large numbers for weighted sums of fuzzy random variables.

Kramosil and Michalek [8] defined the concept of a fuzzy metric space that generalized the probabilistic metric space concept given by Menger [9]. Later, George and Veeramani [10] modified the concept of this space and defined a Hausdorff and first countable topology on the modified fuzzy metric space. This fact has been successfully used in engineering applications such as color image filtering in [11, 12] and perceptual color differences in [13, 14]. In this study, we prove an embedding theorem and generalized Lebesgue convergence and Shapley-Folkman-Starr theorems for fuzzy random variables in fuzzy metric space.

Efron's bootstrapping [15] is a resampling scheme that is used on a variety of estimation problems. Given the importance of the SLLN in the bootstrap method, much has been done in this area by various researchers (see $16[16]$ and 16 et al. [17]). 
In this study, we generalize the SLLN in the fuzzy metric space for the bootstrap mean. In Section 2, some preliminaries and lemmas are presented. In the next section, we introduce the space of fuzzy sets. In Section 4, the generalized Radstrom embedding theorem is expressed. In the next section, Lebesgue dominated convergence and the Shapley-Folkman-Starr theorems will be generalized. The SLLN in fuzzy metric space is given in Section 6 In Section 7, we use the SLLN in the fuzzy metric space for the bootstrap mean. The last section concludes the paper.

\section{Preliminaries}

In this section, we provide the definition of t-norms and the elementary concepts of fuzzy set theory that will be used in the following sections.

Triangular norms (t-norms), introduced by Schweizer and Sklar [18], play a key role in the theory of fuzzy metric spaces.

Definition 1 [19]. A $t$-norm is a binary operation $*$ : [0, 1] $\times$ $[0,1] \rightarrow[0,1]$, such that, for all $a, b, c, d \in[0,1]$, the following four axioms are satisfied:

(i) $a * 1=a$;

(ii) $a * b \leq c * d$ whenever $a \leq c$ and $b \leq d$;

(iii) $a * b=b * a$;

(iv) $a *(b * c)=(a * b) * c$.

A $\mathrm{t}$-norm $*$ is said to be continuous if it is a continuous function on $[0,1] \times[0,1]$.

A fuzzy subset (fuzzy set) of $\mathbb{R}^{p}, p \geq 1$, is a function of $u: \mathbb{R}^{p} \rightarrow[0,1]$. We denote the support of $u$, that is, the closure of $\left\{x \in \mathbb{R}^{p} \mid u(x)>0\right\}$, by supp $u$. For each fuzzy set $u$, the $\alpha$-level set is defined by

$$
L_{\alpha} u=\left\{x \in \mathbb{R}^{p} \mid u(x) \geq \alpha\right\}, 0<\alpha \leq 1 .
$$

Suppose $\mathcal{F}\left(\mathbb{R}^{p}\right)$ is the collection of those fuzzy sets on $\mathbb{R}^{p}$. Two fuzzy sets $u$ and $v$ are equal, written as $u=v$, if and only if $u(x)=v(x)$ for all $x$ in $\mathbb{R}^{p}[20$.

We denote $d(u, v)$ as a metric in $\mathcal{F}\left(\mathbb{R}^{p}\right)$; that is, for $u, v, z \in$ $\mathcal{F}\left(\mathbb{R}^{p}\right)$,

(i) $d(u, v) \geq 0$;

(ii) $d(u, v)=0$ if and only if $u=v$;

(iii) $d(u, z) \leq d(u, v)+d(v, z)$.
In addition, the norm of $u$ is defined as $\|u\|=d\left(u, I_{\{0\}}\right)$, where $I_{\{0\}}$ is an indicator function (the fuzzy set taking value 1 at 0 and 0 for all $x \neq 0$ ).

Let $\mathcal{F}_{c}\left(\mathbb{R}^{p}\right)$ be the collection of the fuzzy sets $u: \mathbb{R}^{p} \rightarrow$ $[0,1]$ with the following properties:

(i) $L_{\alpha} u$ is compact for all $0<\alpha \leq 1$;

(ii) $\operatorname{supp} u$ is compact;

(iii) $\left\{x \in \mathbb{R}^{p} \mid u(x)=1\right\} \neq \emptyset$.

In this case,

$$
d_{r}(u, v)= \begin{cases}\left(\int_{0}^{1} d_{H}^{r}\left(L_{\alpha} u, L_{\alpha} v\right) d \alpha\right)^{\frac{1}{r}}, & r>0, \\ \operatorname{supp}_{\alpha>0} d_{H}\left(L_{\alpha} u, L_{\alpha} v\right), & r=\infty,\end{cases}
$$

is a metric in $\mathcal{F}_{c}\left(\mathbb{R}^{p}\right)$ such that $d_{H}$ is the Pompeiu-Hausdorff distance. There is no unique metric in $\mathcal{F}_{c}\left(\mathbb{R}^{p}\right)$ that extends the Pompeiu-Hausdorff distance [2]. Denote by $\mathcal{F}_{c c}\left(\mathbb{R}^{p}\right)$ the space of fuzzy sets $u \in \mathcal{F}_{c}\left(\mathbb{R}^{p}\right)$ such that $L_{\alpha} u$ for all $\alpha \geq 0$ is convex That is, $u \in \mathcal{F}_{c c}\left(\mathbb{R}^{p}\right)$ if $u \in \mathcal{F}_{c}\left(\mathbb{R}^{p}\right)$ and $u$ is fuzzy convex:

$u(\lambda x+(1-\lambda) y) \geq \min [u(x), u(y)], x, y \in \mathbb{R}^{p}, \lambda \in[0,1]$.

Zadeh's extension principle supplies a linear structure in $\mathcal{F}\left(\mathbb{R}^{p}\right)$ by the operations

$$
\begin{aligned}
& (u \pm v)(x)=\sup \min _{y \pm z=x}[u(y), v(z)], \\
& (\lambda u)(x)= \begin{cases}u\left(\lambda^{-1} x\right), & \lambda \neq 0, \\
I_{\{0\}}, & \lambda=0,\end{cases}
\end{aligned}
$$

where $u, v \in \mathcal{F}\left(\mathbb{R}^{p}\right)$ and $\lambda \in \mathbb{R}$.

Propositions 1,3 will lead to Theorem 3 , which is the desired embedding result.

Proposition $1[21]$. If $u, v \in \mathcal{F}_{c c}\left(\mathbb{R}^{p}\right)$, then

$$
L_{\alpha}(u+v)=L_{\alpha} u+L_{\alpha} v .
$$

Proposition 2 [21]. If $u, v, w \in \mathcal{F}_{c c}\left(\mathbb{R}^{p}\right)$, then $u+w=$ $v+w$ and then $u=v$.

Proposition $3[21]$. If $u, v, w \in \mathcal{F}_{c c}\left(\mathbb{R}^{p}\right)$, then

$$
d_{\infty}(u+w, v+w)=d_{\infty}(u, v) .
$$

Corollary 1. Relation (2) also follows for $d_{r}, r<\infty$.

In addition, in this study, $\mathscr{K}_{c}\left(\mathbb{R}^{p}\right)$ is the collection of a nonempty compact subset of $\mathbb{R}^{p}$ and $\mathscr{K}_{c c}\left(\mathbb{R}^{p}\right)$ denotes the 
nonempty compact convex subsets of $\mathbb{R}^{p}$. The space $\mathscr{K}_{c}\left(\mathbb{R}^{p}\right)$ has a linear structure induced by the Minkowski addition and scalar multiplication:

$$
A+B=\{a+b \mid a \in A, b \in B\}, \lambda A=\{\lambda a \mid a \in A\},
$$

where $A, B \in \mathscr{K}_{c}\left(\mathbb{R}^{p}\right)$ and $\lambda \in \mathbb{R}$. Note that $\mathscr{K}_{c}\left(\mathbb{R}^{p}\right)$ is not a vector space, but it becomes a complete metric space when endowed with the Pompeiu-Hausdorff distance.

If $\lambda=-1$, scalar multiplication gives the opposite: $-A=$ $(-1) A=\{-a \mid a \in A\}$; however, in general, $A+(-A) \neq\{0\}$; that is, the opposite of $A$ is not the inverse of $A$ in Minkowski addition (unless $A=\{a\}$ is a singleton). The Minkowski difference is $A-B=A+(-1) B=\{a-b \mid a \in A, b \in B\}$. In general, even if it holds that $(A+C=B+C) \Longleftrightarrow A=$ $B$, addition and subtraction simplification is not valid; that is, $(A+B)-B \neq A$.

To partially overcome this situation, Hukuhara [22] introduced the following H-difference (for all $A, B \in \mathscr{K}_{c c}\left(\mathbb{R}^{p}\right)$ ):

$$
A \ominus_{H} B=C \Longleftrightarrow A=B+C,
$$

and an important property of $\ominus_{H}$ is that $A \ominus_{H} A=\{0\}$. From an algebraic point of view, the difference between the two sets $A$ and $B$ may be interpreted either in terms of addition as in Eq. (3) or in terms of negative addition, i.e.,

$$
A \boxminus B=C \Longleftrightarrow B=A+(-1) C,
$$

where $(-1) C$ is the opposite set of $C$. Conditions Eqs. (3) and (4) are compatible with each other, which suggests a generalization of the Hukuhara difference [23]:

Definition 2 [23]. Let $\mathcal{X}$ be a vector space with the induced topology and $A, B \in \mathscr{K}_{c}(\mathcal{X})$. We define the generalized Hukuhara difference (gH-difference) of $A$ and $B$ as the set $C \in \mathscr{K}_{c}(\mathcal{X})$ such that

$$
A \ominus_{g} B=C \Longleftrightarrow\left\{\begin{aligned}
(i) & A=B+C \\
\text { or } & (i i) \quad B=A+(-1) C .
\end{aligned}\right.
$$

In the fuzzy or interval arithmetic context, equation $u=$ $v+w$ is not equivalent to $w=u-v=u+(-1) v$ nor to $v=u-w=u+(-1) w$. This has motivated the introduction of the following gH-difference for fuzzy numbers [24, 25]:

Definition 3 [23]. Given $u, v \in \mathcal{F}_{c c}\left(\mathbb{R}^{p}\right)$, the gH-difference is the fuzzy number $w \in \mathcal{F}_{c c}\left(\mathbb{R}^{p}\right)$, if it exists, such that

$$
u \ominus_{g} v=w \Longleftrightarrow\left\{\begin{array}{cl}
(i) & u=v+w \\
\text { or }(i i) & v=u+(-1) w .
\end{array}\right.
$$

Proposition 4 [23]. Let $u, v \in \mathcal{F}_{c c}\left(\mathbb{R}^{p}\right)$. The gH-difference $\ominus_{g}$ exists; it is unique and has the following properties (where $I_{\{0\}}$ denotes the crisp set $\left.\{0\}\right)$ :

(i) $u \ominus_{g} u=I_{\{0\}}$.

(ii) (a) $(u+v) \ominus_{g} v=u$; (b) $u \ominus_{g}(u-v)=v$.

(iii) If $u \ominus_{g} v$ exists, then $(-v) \ominus_{g}(-u)$ also exists and $I_{\{0\}} \ominus_{g}$ $\left(u \ominus_{g} v\right)=(-v) \ominus_{g}(-u)$.

(iv) $u \ominus_{g} v=v \ominus_{g} u=w$ if and only if $w=-w$ (in particular, $w=I_{\{0\}}$ if and only if $u=v$ ).

(v) If $v \ominus_{g} u$ exists, then either $u+\left(v \ominus_{g} u\right)=v$ or $v-\left(v \ominus_{g}\right.$ $u)=u$ and if both equalities hold then $v \ominus_{g} u$ is a crisp set.

\section{Fuzzy Metric Spaces for Fuzzy Sets}

Distance measures are one of the most important tools in diverse fields such as remote sensing, data mining, pattern recognition, and multivariate data analysis. Several distance measures for precise numbers are well established in the literature. Owing to the existence of vagueness, a logical problem arises as the distance is computed in an imprecise framework. In these cases, the crisp number is transformed into a fuzzy number.

Many authors have introduced the concept of fuzzy metric spaces in different ways [26-28]. The 3-tuple $(\mathscr{X}, M, *)$ is said to be a fuzzy metric space whenever $M$ is a fuzzy set (fuzzy metric) on $\mathscr{X} \times \mathscr{X} \times(0, \infty), \mathscr{X}$ is an arbitrary nonempty set, and $*$ is a continuous t-norm [10]. A sequence $\left\{x_{n}\right\}$ in a fuzzy metric space $(\mathscr{X}, M, *)$ is a Cauchy sequence if and only if, for each $0<\varepsilon<1$ and $t>0$, there exists $n_{0} \in \mathbb{N}$ such that for all $n, m \geq n_{0}$

$$
M\left(x_{n}, x_{m}, t\right)>1-\varepsilon .
$$

A fuzzy metric space is said to be complete if and only if every Cauchy sequence is convergent [10].

Saadati and Vaezpour [29] defined fuzzy normed spaces. The 3-tuple $(\mathcal{X}, N, *)$ is said to be a fuzzy normed space if $\mathcal{X}$ is a vector space, $*$ is a continuous t-norm, and $N$ is a fuzzy set (fuzzy norm) on $\mathcal{X} \times(0, \infty)$. 
One of the most important applications of fuzzy metrics is noise detection in image processing. Morillas et al. [30] used a fuzzy metric to detect noise and showed that this method is better than other methods. In fact, Morillas et al. [30] used the following standard fuzzy metric:

$$
R\left(F_{i}, F_{j}, t\right)=\frac{t}{t+\left\|F_{i}-F_{j}\right\|},
$$

where $F_{i}=\left(F_{i}^{1}, F_{i}^{2}, F_{i}^{3}\right)$ are $\mathrm{R}$ (red), $\mathrm{G}$ (green), and $\mathrm{B}$ (blue) for each pixel of the image. Now, if we look at the colors with fuzzy vision, we cannot use the above method. Therefore, it is necessary to use the definition of the fuzzy metric for fuzzy sets.

In the following, by using the definition of the fuzzy metric space in [10] and the fuzzy normed space in [29], we define the fuzzy metric space and the fuzzy normed space for fuzzy sets.

Definition 4. Let $\mathscr{X}$ be an arbitrary nonempty set, and let $\mathcal{F}(\mathscr{X})$ be the collection of those fuzzy sets on $\mathscr{X}$, and define * as a continuous t-norm. The 3 -tuple $\left(\mathcal{F}(\mathscr{X}), M^{\prime}, *\right)$ is said to be a fuzzy metric space for fuzzy sets if $M^{\prime}$ is a fuzzy set (fuzzy metric for fuzzy sets) on $\mathcal{F}(\mathscr{X}) \times \mathcal{F}(\mathscr{X}) \times(0, \infty)$ satisfying the following conditions for all $u, v, z \in \mathcal{F}(\mathscr{X})$ and $t, s>0$ :

(i) $M^{\prime}(u, v, 0)>0$;

(ii) $M^{\prime}(u, v, t)=1$ for all $t>0$ if and only if $u=v$;

(iii) $M^{\prime}(u, v, t)=M^{\prime}(v, u, t)$;

(iv) $M^{\prime}(u, v, t) * M^{\prime}(v, z, s) \leq M^{\prime}(u, z, t+s)$;

(v) $M^{\prime}(u, v,):.(0, \infty) \rightarrow[0,1]$ is continuous.

Example 1. Let $\left(\mathcal{F}(\mathscr{X}), M^{\prime}, *\right)$ be a fuzzy metric space for fuzzy sets. Define $a * b=a b$ or $a * b=\min \{a, b\}$ and $\forall u, v \in \mathcal{F}(\mathscr{X})$. Then,

$$
M^{\prime}(u, v, t)=\frac{k t^{n}}{k t^{n}+m d(u, v)}, \quad k, m \in \mathbb{R}^{+}, n \geq 1 .
$$

It is easy to show that $\left(\mathcal{F}(\mathscr{X}), M^{\prime}, *\right)$ is a fuzzy metric space for fuzzy sets. In particular, if $k=n=m=1$, then

$$
M^{\prime}(u, v, t)=\frac{t}{t+d(u, v)},
$$

which is called the standard fuzzy metric for fuzzy sets.

Definition 5. Let $\mathcal{X}$ be a vector space and $\mathcal{F}(\mathcal{X})$ be the collection of these fuzzy sets on $\mathcal{X}$. The 3 -tuple $\left(\mathcal{F}(\mathcal{X}), N^{\prime}, *\right)$ is said to be a fuzzy normed space if $*$ is a continuous t-norm and $N^{\prime}$ is a fuzzy set (fuzzy norm for fuzzy sets) on $\mathcal{F}(\mathcal{X}) \times$ $(0, \infty)$ satisfying the following conditions for every $u, v \in$ $\mathcal{F}(\mathcal{X})$ and $t, s>0$ :

(i) $N^{\prime}(u, t)>0$;

(ii) $N^{\prime}(u, t)=1$ if and only if $u=I_{\{0\}}$;

(iii) $N^{\prime}(\alpha u, t)=N^{\prime}(u, t /|\alpha|)$, for all $\alpha \neq 0$;

(iv) $N^{\prime}(u, t) * N^{\prime}(v, s) \leq N^{\prime}(u+v, t+s)$;

(v) $N^{\prime}(u,):.(0, \infty) \rightarrow[0,1]$ is continuous;

(vi) $\lim _{t \rightarrow \infty} N^{\prime}(u, t)=1$.

Example 2. Let $(\mathcal{X},\|\cdot\|)$ be a normed space. We define $a * b=a b$ and

$$
N^{\prime}(u, t)=\left(\exp \frac{\|u\|}{t}\right)^{-1},
$$

for $u \in \mathcal{F}(\mathcal{X})$ and $t \in(0, \infty)$. Then, $\left(\mathcal{F}(\mathcal{X}), N^{\prime}, *\right)$ is a fuzzy normed space.

Definition 6. Let $\left(\mathcal{F}(\mathcal{X}), N^{\prime}, *\right)$ be a fuzzy normed space for fuzzy sets and $N^{\prime}(u, t)=\varphi(\|u\|, t)$ be the fuzzy norm for fuzzy sets, where $\varphi(., t)$ is a decreasing function and $\|\cdot\|=$ $d\left(., I_{\{0\}}\right)$; then, we call it the fuzzy norm induced by $d$ and indicate it by $N_{d}^{\prime}$.

Example 3. Let $\left(\mathcal{F}(\mathcal{X}), N^{\prime}, *\right)$ be a fuzzy normed space for fuzzy sets and let $a * b=a b$ or $a * b=\min \{a, b\}$ and $\forall u, v \in \mathcal{F}(\mathcal{X})$. In this case,

$$
N^{\prime}(u, t)=\frac{k t^{n}}{k t^{n}+m\|u\|}, \quad k, m \in \mathbb{R}^{+}, n \geq 1,
$$

is a fuzzy norm for fuzzy sets. Given that $\|u\|=d\left(u, I_{\{0\}}\right)$, we can write

$$
N^{\prime}(u, t)=\frac{k t^{n}}{k t^{n}+m d\left(u, I_{\{0\}}\right)} .
$$

The conditions of Definition 5 for $N^{\prime}(u, t)$ follow, and $(\mathcal{F}(\mathcal{X})$, $\left.N^{\prime}, *\right)$ is a fuzzy normed space for fuzzy sets. In particular, if $k=n=m=1$, then

$$
N^{\prime}(u, t)=\frac{t}{t+d\left(u, I_{\{0\}}\right)} .
$$

which is called the standard fuzzy norm for fuzzy sets.

Lemma 1. Let $N^{\prime}$ be a fuzzy norm for fuzzy sets. Then

$$
N^{\prime}\left(u \ominus_{g} v, t\right)=N^{\prime}\left(v \ominus_{g} u, t\right) .
$$


Proof. According to Proposition 4 and Definition 5, Eq. (9) is satisfied.

Theorem 1. Let $\left(\mathcal{F}_{c c}\left(\mathbb{R}^{p}\right), N^{\prime}, *\right)$ be a fuzzy normed space for fuzzy sets. If we define

$$
M_{d}^{\prime}(u, v, t)=N_{d}^{\prime}\left(u \ominus_{g} v, t\right)
$$

where $u, v \in \mathcal{F}_{c c}\left(\mathbb{R}^{p}\right), t>0$, and $\ominus_{g}$ is the gH-difference, then $M_{d}^{\prime}$ is a fuzzy metric for fuzzy sets in $\mathcal{F}_{c c}\left(\mathbb{R}^{p}\right)$, which is called the fuzzy metric induced by the fuzzy norm $N_{d}^{\prime}$.

Proof. Let $u, v, z \in \mathcal{F}_{c c}\left(\mathbb{R}^{p}\right)$; then

$$
L_{\alpha} u, L_{\alpha} v, L_{\alpha} z \in \mathscr{K}_{c c}\left(\mathbb{R}^{p}\right) \text {. }
$$

By using the gH-difference, we show that the conditions of Definition 4 are established:

1. $M_{d}^{\prime}(u, v, t)=N_{d}^{\prime}\left(u \ominus_{g} v, t\right)>0$;

2. $u=v$ if and only if $M_{d}^{\prime}(u, v, t)=N_{d}^{\prime}\left(u \ominus_{g} v, t\right)=$ $N_{d}^{\prime}\left(I_{\{0\}}, t\right)=1$.

3.

$$
\begin{aligned}
M_{d}^{\prime}(u, v, t) & =N_{d}^{\prime}\left(u \ominus_{g} v, t\right) \\
& =N_{d}^{\prime}\left((-1)\left(v \ominus_{g} u\right), t\right) \\
& =N_{d}^{\prime}\left(v \ominus_{g} u, \frac{t}{|-1|}\right) \\
& =M_{d}^{\prime}(v, u, t) ;
\end{aligned}
$$

4. for all $u, v, z \in \mathcal{F}_{c c}\left(\mathbb{R}^{p}\right)$ and $t, s \in \mathbb{R}^{+}$,

$$
\begin{aligned}
M_{d}^{\prime}(u, v, t) * M_{d}^{\prime}(v, z, s)= & N_{d}^{\prime}\left(u \ominus_{g} v, t\right) \\
& * N_{d}^{\prime}\left(v \ominus_{g} z, s\right), \\
\leq & N_{d}^{\prime}\left(u \ominus_{g} z, t+s\right) \\
= & M_{d}^{\prime}(u, z, t+s) ;
\end{aligned}
$$

5. $M_{d}^{\prime}(u, v,)=.N_{d}^{\prime}\left(u \ominus_{g} v,.\right):(0, \infty) \rightarrow[0,1]$ is continuous.

Corollary 2. Let $\left(\mathcal{F}_{c c}\left(\mathbb{R}^{p}\right), N_{d_{1}}^{\prime}, *\right)$ be a fuzzy normed space for fuzzy sets and let $M_{d_{1}}^{\prime}$ be a fuzzy metric induced by $N_{d_{1}}^{\prime}$; then $\left(\mathcal{F}_{c c}\left(\mathbb{R}^{p}\right), M_{d_{1}}^{\prime}, *\right)$ is a fuzzy metric space.

Lemma 2. A fuzzy metric for fuzzy sets $M_{d}^{\prime}$ that is induced by a fuzzy norm $N_{d}^{\prime}$ has the following properties for all $u, v, z \in \mathcal{F}(\mathcal{X})$ and every scalar $\alpha \neq 0$ :

(i) $M_{d}^{\prime}(u+z, v+z, t)=M_{d}^{\prime}(u, v, t)$; (ii) $M_{d}^{\prime}(\alpha u, \alpha v, t)=M_{d}^{\prime}(u, v, t /|\alpha|)$.

Proof. We can simply proof this lemma by using Lemma 4 in [29] and Definition 5. For example, for $(i i)$, we have that

$$
\begin{aligned}
M_{d}^{\prime}(\alpha u, \alpha v, t) & =N_{d}^{\prime}\left(\alpha u \ominus_{g} \alpha v, t\right) \\
& =N_{d}^{\prime}\left(u \ominus_{g} v, t /|\alpha|\right) \\
& =M_{d}^{\prime}(u, v, t /|\alpha|) .
\end{aligned}
$$

In the following, we present the separability of $\mathcal{F}_{c c}\left(\mathbb{R}^{p}\right)$ in the fuzzy metric $\left(\mathcal{F}_{c c}\left(\mathbb{R}^{p}\right), M_{d_{r}}^{\prime}, *\right)$ for $r=1$.

Theorem 2. Fuzzy metric space $\left(\mathcal{F}_{c c}\left(\mathbb{R}^{p}\right), M_{d_{1}}^{\prime}, *\right)$ is separable.

Proof. We know that $\left(\mathcal{F}_{c}\left(\mathbb{R}^{p}\right), d_{1}\right)$ is a separable metric space [2]. It follows easily that $\left(\mathcal{F}_{c c}\left(\mathbb{R}^{p}\right), d_{1}\right)$ is a separable metric space and, given that the continuous image of a separable metric space is separable, the result is established.

Because the compact subsets in the Banach space are not a vector space (with respect to Minkowski addition), Radstrom [31] showed that a class of convex compact sets, represented by $\operatorname{co} \mathscr{K}_{c}\left(\mathbb{R}^{p}\right)$ or $\mathscr{K}_{c c}\left(\mathbb{R}^{p}\right)$, can be embedded isometrically into normed space. In addition, the spaces of the fuzzy sets are not vector spaces. In the next section, we generalize the Radstrom embedding theorem for fuzzy sets.

\section{Generalized Radstrom Embedding Theorem}

Klement et al. [2] showed that $\mathcal{F}_{c c}\left(\mathbb{R}^{p}\right)$ can be embedded isometrically into a normed space. Here, we show that a class of fuzzy convex sets that is represented by $\mathcal{F}_{c c}\left(\mathbb{R}^{p}\right)$ can be embedded isometrically into a fuzzy normed space. In fact, we introduce a new embedding theorem for fuzzy compact convex sets. This theorem enables us to prove the SLLN.

The space $\mathcal{F}_{c c}\left(\mathbb{R}^{p}\right)$ plays an important role because it can be embedded isometrically into a fuzzy normed space. This embedding generalizes the Radstrom embedding theorem [31] from $\mathcal{F}_{c c}\left(\mathbb{R}^{p}\right)$ into a fuzzy normed space.

Theorem 3. Let $u, v \in \mathcal{F}_{c c}\left(\mathbb{R}^{p}\right)$. There exist a fuzzy normed space $\chi$ and a function $j: \mathcal{F}_{c c}\left(\mathbb{R}^{p}\right) \rightarrow \chi$ with the following properties:

1. $M_{d_{1}}^{\prime}(j(u), j(v), t)=M_{d_{1}}^{\prime}(u, v, t)$.

2. $j(u+v)=j(u)+j(v)$.

3. $j(\lambda u)=\lambda j(u), \lambda \geq 0$. 
Proof. By using Propositions 1 and 2 and [31], conditions 1-7 of the Radstrom embedding theorem are established.

Conditions 8 and 9 follow by Proposition 3 in $d_{1}$. In addition, given that $M_{d_{1}}^{\prime}$ is a decreasing and continuous function of $d_{1}$, conditions 8 and 9 follow in $M_{d_{1}}^{\prime}$.

According to Theorem $2[21]$ and the continuity of $M_{d_{1}}^{\prime}$ with respect to $d_{1}$, condition 10 of the Radstrom embedding theorem [31] follows.

Properties 2 and 3 follow from the definitions.

\section{Generalized Lebesgue Convergence and Shapley-Folkman-Starr Theorems}

Two important tools in Section 6, which are used to prove the SLLN for fuzzy random variables in fuzzy metric space, are Lebesgue convergence and the Shapley-Folkman-Starr theorems. In this section, after defining the random closed set, random compact set, and fuzzy random variables, we generalize these theorems for fuzzy random variables in the fuzzy metric space.

Suppose that $(\Omega, \mathcal{A}, P)$ is a probability space. The following definition describes the concept of a random closed set and a random compact set.

Definition 7 [32]. Let $C\left(\mathbb{R}^{p}\right)$ be the family of closed subsets of $\mathbb{R}^{p}$. A map $\mathfrak{X}: \Omega \rightarrow C\left(\mathbb{R}^{p}\right)$ is called a random closed set if, for every compact set $K \in \mathscr{K}_{c}\left(\mathbb{R}^{p}\right)$,

$$
\{\omega: \mathfrak{X} \cap K \neq \emptyset\} \in \mathcal{A} .
$$

Definition 8 [32]. A random closed set $\mathfrak{X}$ with almost surely compact values (such that $\mathfrak{X} \in \mathscr{K}_{c}\left(\mathbb{R}^{p}\right)$ a.s.) is called a random compact set.

The $\mathbb{R}^{p}$-valued random set (i.e., random sets whose values are compact subsets of $\mathbb{R}^{p}$ ) is a Borel measurable function $\mathfrak{X}: \Omega \rightarrow \mathscr{K}_{c}\left(\mathbb{R}^{p}\right)$.

Definition $9[32]$. Let $\mathfrak{B}\left(\mathbb{R}^{p}\right)$ be the Borel $\sigma$-algebra. Random closed sets $\mathfrak{X}_{1}, \ldots, \mathfrak{X}_{n}$ are said to be independent if

$P\left(\mathfrak{X}_{1} \in A_{1}, \ldots, \mathfrak{X}_{n} \in A_{n}\right)=P\left(\mathfrak{X}_{1} \in A_{1}\right) \cdot \ldots \cdot P\left(\mathfrak{X}_{n} \in A_{n}\right)$

for all $A_{1}, \ldots, A_{n} \in \mathfrak{B}\left(\mathbb{R}^{p}\right)$.

For more information about this concept, see [32]. A random closed set $\mathfrak{X}$ in the Euclidean space $\mathbb{R}^{p}$ is integrably bounded if

$$
\|\mathfrak{X}\|=\sup \{\|x\| \mid x \in \mathfrak{X}\}
$$

has a finite expectation [32]. That is, $E\|\mathfrak{X}\|<\infty$. The expected value of the random set was defined by Aumann [33] and later by Debreu [34]. These definitions were shown to be equivalent by Byrne [35]. If $\mathfrak{X}$ is a random compact set, then $E \mathfrak{X}$ is defined as

$$
E \mathfrak{X}=\left\{E f ; f \in L^{1}(\Omega, \mathcal{A}, P), f(\omega) \in \mathfrak{X}(\omega) \text { a.e. }\right\} .
$$

where $f: \Omega \rightarrow \mathbb{R}^{p}$ is a selection of $\mathfrak{X}$ and $E f$ denotes the classical expectation (via the Bochner integral). In general, $E \mathfrak{X}$ may be empty, but if $E\|\mathfrak{X}\|<\infty$, then $E \mathfrak{X} \in \mathscr{K}_{c}\left(\mathbb{R}^{p}\right)$ [33, 34]

Definition 10 [1]. A fuzzy random variable is a function $X: \Omega \rightarrow \mathcal{F}_{c}\left(\mathbb{R}^{p}\right)$ such that

$$
\left\{(\omega, x): x \in L_{\alpha} X(\omega)\right\} \in \mathcal{A} \times \mathcal{B}
$$

for $\alpha \in[0,1]$, where $L_{\alpha} X: \Omega \rightarrow \mathscr{K}_{c}\left(\mathbb{R}^{p}\right)$ is defined by

$$
L_{\alpha} X(\omega)=\left\{X \in \mathbb{R}^{p}: \mu_{X(\omega)}(x) \geq \alpha\right\} .
$$

Fuzzy random variable $X$ is integrable in $d_{1}$ metric whenever

$$
E\|\operatorname{supp} X\|<\infty \text {. }
$$

Definition $11[6]$. Let $X, Y$ be fuzzy random variables. $X, Y$ is called identical if, for any $\alpha \in[0,1], L_{\alpha} X(\omega)=L_{\alpha} Y(\omega)$ a.e.

Definition 12 [6]. A sequence of fuzzy random variables $\left\{X^{p}: p \in \mathbb{N}\right\}$ is called independent if, for any $\alpha \in[0,1]$, the sequence of random sets $\left\{L_{\alpha} X^{p}: p \in \mathbb{N}\right\}$ is independent.

The convergence of fuzzy random variables is usually defined with respect to the generalization of the Pompeiu-Hausdorff metric as $d_{1}\left(X_{n}, X\right) \rightarrow 0$ [1]. It can be said that $X_{n} \rightarrow X$ a.e. in fuzzy metric $M_{d_{1}}^{\prime}$ whenever $M_{d_{1}}^{\prime}\left(X_{n}, X, t\right) \rightarrow 1$ a.e.

Theorem 4. Let $\left\{X_{k} \mid k \geq 1\right\}$, where $X$ is a fuzzy random variable with values in $\mathcal{F}_{c c}\left(\mathbb{R}^{p}\right)$ such that $E\left\|\operatorname{supp} X_{k}\right\|<\infty$ and $E\|\operatorname{supp} X\|<\infty$. If $X_{k}$ in the fuzzy metric $M_{d_{1}}^{\prime}$ is convergent a.e. to $X$ and $d_{1}\left(X_{k}(\omega), I_{\{0\}}\right) \leq h(\omega)$ for all $k \geq 1$, where $h: \Omega \rightarrow \mathbb{R}$ is integrable, then, in the fuzzy metric $M_{d_{1}}^{\prime}$,

$$
E X_{k} \rightarrow E X
$$

Proof. Using the inequality in Debreu [34, pp. 366-367] and [2], we have

$$
d_{1}\left(E X_{k}, E X\right) \leq E d_{1}\left(X_{k}, X\right)
$$


And we know that $d_{1}\left(X_{k}, X\right)$ is a random variable; therefore, $E d_{1}\left(X_{k}, X\right)$ is real. Therefore, according to Definition 6 and Theorem 1, we have

$$
M_{d_{1}}^{\prime}\left(E X_{k}, E X, t\right) \geq M_{E d_{1}}^{\prime}\left(X_{k}, X, t\right)
$$

However, from the hypothesis, because $M_{d_{1}}^{\prime}\left(X_{k}, X\right) \rightarrow 1$ a.e., then $d_{1}\left(X_{k}, X\right) \rightarrow 0$ a.e. By using the triangle inequality, we obtain

$$
\begin{aligned}
d_{1}\left(X_{k}, X\right) & \leq d_{1}\left(X_{k}, I_{\{0\}}\right)+d_{1}\left(X, I_{\{0\}}\right) \\
& \leq h+d_{1}\left(X, I_{\{0\}}\right) .
\end{aligned}
$$

According to the integrability of $h$ and $X \in \mathcal{F}_{c c}\left(\mathbb{R}^{p}\right), d_{1}\left(X_{k}, X\right)$ is integrable. Now, from the Lebesgue dominated convergence theorem,

$$
E d_{1}\left(X_{k}, X\right) \rightarrow 0
$$

Therefore,

$$
M_{E d_{1}}^{\prime}\left(X_{k}, X, t\right) \rightarrow 1
$$

Furthermore, given Eq. (13), it follows that $d_{1}\left(E X_{k}, E X\right) \rightarrow$ 0. Also, from Eq. 14 we conclude that

$$
M_{d_{1}}^{\prime}\left(E X_{k}, E X, t\right) \rightarrow 1
$$

This means that Eq. (12) holds.

The Shapley-Folkman theorem (see [36]) can be used to show that addition of sets is, in some sense, "convexifying": For any $\mathfrak{X}_{1}, \ldots, \mathfrak{X}_{n} \in \mathscr{K}_{c}\left(\mathbb{R}^{p}\right)$, we have

$$
d\left[\sum_{k=1}^{n} \mathfrak{X}_{k}, \sum_{k=1}^{n} \text { co } \mathfrak{X}_{k}\right] \leq \sqrt{p} \max _{1 \leq k \leq n}\left\|\mathfrak{X}_{k}\right\|,
$$

where $p$ is the dimension of $\mathfrak{X}$ and $p<\infty$ [37. In this study, we generalize the Shapley-Folkman-Starr theorem for fuzzy random variables in fuzzy metric space.

Theorem 5. Suppose $X_{1}, \ldots, X_{n} \in \mathcal{F}_{c}\left(\mathbb{R}^{p}\right), n \in N$. For fuzzy random variables in fuzzy metric space,

$$
\begin{aligned}
& M_{d_{\infty}}^{\prime}\left(\sum_{k=1}^{n} X_{k}, \sum_{k=1}^{n} \operatorname{co} X_{k}, t\right) \\
& \geq \min _{1 \leq k \leq n} M_{d_{\infty}}^{\prime}\left(X_{k}, I_{\{0\}}, \frac{t}{\sqrt{p}}\right),
\end{aligned}
$$

where $I_{\{0\}}$ is a characteristic function (such that, for each $A \in$ $\mathscr{K}_{c}\left(\mathbb{R}^{p}\right)$, its characteristic function $\left.I_{\{A\}} \in \mathcal{F}_{c}\left(\mathbb{R}^{p}\right)\right)$.

Proof. From the Shapley-Folkman theorem in [36], we know

$$
\begin{aligned}
d_{H}\left[L_{\alpha} \sum_{k=1}^{n} X_{k}, L_{\alpha} \sum_{k=1}^{n} \operatorname{co} X_{k}\right] & \leq \sqrt{p} \max _{1 \leq k \leq n}\left\|L_{\alpha} X_{k}\right\| \\
& =\sqrt{p} \max _{1 \leq k \leq n} d_{H}\left(L_{\alpha} X_{k},\{0\}\right)
\end{aligned}
$$

for every $\alpha>0$. This implies immediately that

$$
\begin{aligned}
d_{\infty}\left[\sum_{k=1}^{n} X_{k}, \sum_{k=1}^{n} \operatorname{co} X_{k}\right] & \leq \sqrt{p} \max _{1 \leq k \leq n} d_{\infty}\left(X_{k}, I_{\{0\}}\right) \\
& =\sqrt{p} \max _{1 \leq k \leq n}\left\|\operatorname{supp} X_{k}\right\| .
\end{aligned}
$$

For a continuous decreasing function $\varphi\left(d_{\infty}, t\right)$ (with respect to $d_{\infty}$ ) and constant $t$ and characteristics of the Pompeiu-Hausdorff metric,

$$
\begin{aligned}
& \varphi\left(d_{\infty}\left[\sum_{k=1}^{n} X_{k}, \sum_{k=1}^{n} \operatorname{co} X_{k}\right], t\right) \\
& \geq \varphi\left(\sqrt{p} \max _{1 \leq k \leq n} d_{\infty}\left(X_{k}, I_{\{0\}}\right), t\right) \\
& =\min _{1 \leq k \leq n} \varphi\left(\sqrt{p} d_{\infty}\left(X_{k}, I_{\{0\}}\right), t\right) .
\end{aligned}
$$

Then, from Theorem 1 and Lemma 2

$$
\begin{aligned}
& M_{d_{\infty}}^{\prime}\left(\sum_{k=1}^{n} X_{k}, \sum_{k=1}^{n} \operatorname{co~} X_{k}, t\right) \\
& \geq \min _{1 \leq k \leq n} M_{d_{\infty}}^{\prime}\left(\sqrt{p} X_{k}, \sqrt{p} I_{\{0\}}, t\right) \\
& =\min _{1 \leq k \leq n} M_{d_{\infty}}^{\prime}\left(X_{k}, I_{\{0\}}, \frac{t}{\sqrt{p}}\right) .
\end{aligned}
$$

\section{SLLS in Fuzzy Metric Space}

In this section, by using the Radstrom embedding theorem, we establish the SLLN for fuzzy random variables in fuzzy metric space.

Theorem 6. Let $\left\{X_{k} \mid k \geq 1\right\}$ be an i.i.d. fuzzy random variable such that $X_{1}$ is integrable. Then

$$
\left(\sum_{k=1}^{n} X_{k}\right) / n \rightarrow E\left(\operatorname{co} X_{1}\right) \text { a.e. }
$$

With the convergence being in the fuzzy metric $M_{d_{1}}^{\prime}$.

Proof. Let $X_{k}: \Omega \rightarrow \mathcal{F}_{c}\left(\mathbb{R}^{p}\right)$ for $k=1, \ldots, n$ be i.i.d. fuzzy random variables. In addition, co $X_{k}: \Omega \rightarrow \mathcal{F}_{c c}\left(\mathbb{R}^{p}\right)$. Because 
$\mathscr{K}_{c c}\left(\mathbb{R}^{p}\right) \subseteq \mathscr{K}_{c}\left(\mathbb{R}^{p}\right)$ and from Definition $12,\left\{\right.$ co $\left.X_{k} \mid k \geq 1\right\}$ is i.i.d as well.

Consider first $X_{k}: \Omega \rightarrow \mathcal{F}_{c c}\left(\mathbb{R}^{p}\right)$ and let $j: \mathcal{F}_{c c}\left(\mathbb{R}^{p}\right) \rightarrow \chi$ be the isometry provided by the Radstrom embedding theorem. Because $\left(\mathcal{F}_{c c}\left(\mathbb{R}^{p}\right), M_{d_{1}}^{\prime}, *\right)$ is separable (Theorem 2, it is easy to show that the fuzzy normed space $\chi$ is separable. Then $\left\{j\left(X_{k}\right) \mid k \geq 1\right\}$ are i.i.d. $\chi$-valued random elements. By a standard SLLN in $\mathbb{R}^{p}$, it follows that

$$
\frac{1}{n} \sum_{k=1}^{n} j\left(X_{k}\right) \rightarrow E\left(j\left(X_{1}\right)\right) \text { a.e. }
$$

The main point now is to show that

$$
E\left(j\left(\operatorname{co~} X_{1}\right)\right)=j\left(E\left(\operatorname{co} X_{1}\right),\right.
$$

if $c o X_{1}$ is integrable.

First, assume that co $X_{1}$ is a simple function (for some $l$ ), that is, co $X_{1}=\sum_{i=1}^{l} u_{i} I_{A_{i}}$, where $A_{i} \in \mathcal{A}, u_{i} \in \mathcal{F}_{c c}\left(\mathbb{R}^{p}\right)$.

It is easy to check that $E\left(j\left(\right.\right.$ co $\left.\left.X_{1}\right)\right)=j\left(E\left(\right.\right.$ co $\left.\left.X_{1}\right)\right)$ in this case. To prove this, from Theorem 3 , we see that

$$
\begin{aligned}
E\left(j\left(\operatorname{co} X_{1}\right)\right) & =E\left(j\left(\sum_{i=1}^{l} u_{i} I_{A_{i}}\right)\right) \\
& =E\left(\sum_{i=1}^{l} j\left(u_{i}\right) I_{A_{i}}\right)=\sum_{i=1}^{l} j\left(u_{i}\right) P\left(A_{i}\right) \\
& =j\left(\sum_{i=1}^{l} u_{i} P\left(A_{i}\right)\right)=j E\left(\sum_{i=1}^{l} u_{i} I_{A_{i}}\right) \\
& =j\left(E\left(c o X_{1}\right)\right) .
\end{aligned}
$$

Because co $X_{1}$ is measurable, there exists a sequence of simple functions $S_{m}$ with $S_{m} \rightarrow$ co $X_{1}$ a.e. in the fuzzy metric $M_{d_{1}}^{\prime}$. Furthermore, from the continuity of $M_{d_{1}}^{\prime}$ a.e.,

$$
M_{d_{1}}^{\prime}\left(S_{m}, I_{\{0\}}, t\right) \rightarrow M_{d_{1}}^{\prime}\left(c o X_{1}, I_{\{0\}}, t\right) .
$$

We now consider the truncated fuzzy random variable $t_{m}$ as follows $(m \rightarrow \infty)$ :

$$
t_{m}(\omega)=\left\{\begin{array}{cc}
S_{m}(\omega), & M_{d_{1}}^{\prime}\left(S_{m}(\omega), I_{\{0\}}, t\right) \\
& \geq M_{d_{1}}^{\prime}\left(\operatorname{co~} X_{1}(\omega), I_{\{0\}}, \frac{t}{2}\right), \\
I_{\{0\}}, & \text { o.w. }
\end{array}\right.
$$

Note that $t_{m}$ is a simple function. It is easy to see in fuzzy metric $M_{d_{1}}^{\prime}, t_{m} \rightarrow c o X_{1}$, a.e., that

$$
M_{d_{1}}^{\prime}\left(t_{m}(\omega), I_{\{0\}}, t\right) \geq M_{d_{1}}^{\prime}\left(\operatorname{co} X_{1}(\omega), I_{\{0\}}, \frac{t}{2}\right) .
$$

The hypotheses of Theorem 4 are satisfied because, as $t_{m} \rightarrow$ co $X_{1}, E t_{m} \rightarrow E\left(\right.$ co $\left.X_{1}\right)$. Therefore, in $\chi$,

$$
j\left(E t_{m}\right) \rightarrow j\left(E\left(\operatorname{co~} X_{1}\right)\right) .
$$

It is easy to see that $j\left(t_{m}\right) \rightarrow j\left(c o X_{1}\right)$ and, from properties of the Bochner integral, that

$$
E\left(j\left(t_{m}\right)\right) \rightarrow E\left(j\left(\operatorname{co} X_{1}\right)\right) .
$$

Because $j\left(E t_{m}\right)=E\left(j\left(t_{m}\right)\right)$, it follows that

$$
j\left(E\left(\operatorname{co} X_{1}\right)\right)=E\left(j\left(\operatorname{co} X_{1}\right)\right) .
$$

Therefore,

$$
M_{d_{1}}^{\prime}\left(\frac{1}{n} \sum_{k=1}^{n}\left(j\left(\operatorname{co} X_{k}\right) \ominus_{g} j\left(E\left(\operatorname{co} X_{1}\right)\right), I_{\{0\}}, t\right) \rightarrow 1\right. \text { a.e. }
$$

From the properties of $j$, it follows that

$$
M_{d_{1}}^{\prime}\left(\frac{1}{n} \sum_{k=1}^{n} \operatorname{co~} X_{k}, E\left(\operatorname{co~} X_{1}\right), t\right) \rightarrow 1 \text { a.e. }
$$

Theorem 5 gives

$$
\begin{aligned}
& M_{d_{\infty}}^{\prime}\left(\frac{1}{n} \sum_{k=1}^{n} X_{k}, \frac{1}{n} \sum_{k=1}^{n} c o X_{k}, t\right) \\
& \geq \min _{1 \leq k \leq n} M_{d_{\infty}}^{\prime}\left(X_{k}, I_{\{0\}}, \frac{n t}{\sqrt{p}}\right) .
\end{aligned}
$$

Because a.e. $\min _{1 \leq k \leq n} M_{d_{\infty}}^{\prime}\left(X_{k}, I_{\{0\}}, \frac{n t}{\sqrt{p}}\right) \rightarrow 1$, this implies immediately a.e. that

$$
M_{d_{\infty}}^{\prime}\left(\frac{1}{n} \sum_{k=1}^{n} X_{k}, \frac{1}{n} \sum_{k=1}^{n} c o X_{k}, t\right) \rightarrow 1,
$$

and it follows that

$$
M_{d_{1}}^{\prime}\left(\frac{1}{n} \sum_{k=1}^{n} X_{k}, \frac{1}{n} \sum_{k=1}^{n} \operatorname{coX} X_{k}, t\right) \rightarrow 1 .
$$

Now, with respect to the fourth properties in Definition 4 for fuzzy random variables,

$$
M_{d_{1}}^{\prime}\left(\frac{1}{n} \sum_{k=1}^{n} X_{k}, E\left(\operatorname{co} X_{1}\right), t\right)
$$




$$
\begin{aligned}
& \geq M_{d_{1}}^{\prime}\left(\frac{1}{n} \sum_{k=1}^{n} X_{k}, \frac{1}{n} \sum_{k=1}^{n} \operatorname{co} X_{k}, \frac{t}{2}\right) \\
& \quad * M_{d_{1}}^{\prime}\left(\frac{1}{n} \sum_{k=1}^{n} \operatorname{co~} X_{k}, E\left(\operatorname{co} X_{k}\right), \frac{t}{2}\right) .
\end{aligned}
$$

Because the right-side values tend to 1 (according to Eqs. 16) and (17), we have

$$
M_{d_{1}}^{\prime}\left(\frac{1}{n} \sum_{k=1}^{n} X_{k}, E\left(\operatorname{coX} X_{1}\right), t\right) \rightarrow 1,
$$

and the proof is complete.

\section{Bootstrap Mean in Fuzzy Metric Space}

The bootstrap method introduced in Efron [15] is a very general resampling procedure for estimating the distributions of statistics based on independent observations. 16 [16] provided a strong law for the bootstrap, and 16 et al. [17] established laws of large numbers for bootstrapped U-statistics. In addition, Csorgo [38] presented the weak and strong laws of large numbers for bootstrap sample means under minimal moment conditions. In this section, we generalize the SLLN in the fuzzy metric space for the bootstrap mean.

Let $\left\{X_{k} \mid k \geq 1\right\}$ be an infinite sequence of i.i.d. fuzzy random variables defined on a probability space $(\Omega, \mathcal{A}, P)$, and let $X_{1}$ be integrable. For each $n=1,2, \ldots$, let $Y_{n, 1}, Y_{n, 2}$, $\ldots, Y_{n, m(n)}$ be the ordinary Efron bootstrap sample of size $m(n)$, where $\{m(n)\}$ is a sequence of positive integers. The variables $Y_{n, 1}, Y_{n, 2}, \ldots, Y_{n, m(n)}$ result from sampling $m$ times the sequence $\left\{X_{1}, X_{2}, \ldots, X_{n}\right\}$ with replacement such that at each stage any one element has probability $1 / n$ of being selected [38].

Suppose that $\bar{X}_{n}^{*}$ is the bootstrap sample mean where

$$
\bar{X}_{n}^{*}=\frac{1}{m(n)} \sum_{i=1}^{m(n)} Y_{n, i}
$$

Klement et al. [2] provided limit theorems for fuzzy random variables. They showed that

$$
d_{1}\left(\frac{1}{n} \sum_{i=1}^{n} X_{i}, E\left(\operatorname{co} X_{1}\right)\right) \rightarrow 0 \text { a.e. }
$$

Now, if we apply this theorem for the bootstrap mean then we have

$$
d_{1}\left(\frac{1}{m(n)} \sum_{i=1}^{m(n)} Y_{n, i}, E\left(\operatorname{co} X_{1}\right)\right) \rightarrow 0 \text { a.e. }
$$

Sometimes, the expert's opinion may be important in determining the magnitude or smallness of the distance. In this case, the use of the SLLN in the fuzzy metric space (stated in Section 6 is more appropriate. By specifying the value of $t$, the expert can apply his or her opinion on the distance between two values. In the following, we illustrate with an example that the bootstrap SLLN in fuzzy metric space is established. In other words, we show that

$$
M_{d_{1}}^{\prime}\left(\frac{1}{m(n)} \sum_{i=1}^{m(n)} Y_{n, i}, E\left(\operatorname{co} X_{1}\right), t\right) \rightarrow 1 \text { a.e. }
$$

Example 4. Suppose that the profit of selling a piece of property is a triangular fuzzy number. The probability that someone in time $A_{1}$ will sell a piece of property at a profit of $(-0.7,0.3,0.1)_{T}$ thousand dollars is 0.2 . Moreover, the probability of selling at a profit of $(0,0.5,0.5)_{T}$ thousand dollars in time $A_{2}$ is 0.25 , that of selling at a profit of $(1.5,0.25,0.25)_{T}$ in time $A_{3}$ is 0.25 , that of selling at a profit of $(2,0.2,0.4)_{T}$ in time $A_{4}$ is 0.15 , and that of selling at a profit of $(3,0.5,0.5)_{T}$ in time $A_{5}$ is 0.15 . What is the total expected expected profit?

Here we denote the fuzzy random variable with

$$
G(\omega)= \begin{cases}(-0.7,0.3,0.1)_{T}, & \omega \in A_{1}, \\ (0,0.5,0.5)_{T}, & \omega \in A_{2}, \\ (1.5,0.25,0.25)_{T}, & \omega \in A_{3}, \\ (2,0.2,0.4)_{T}, & \omega \in A_{4}, \\ (3,0.5,0.5)_{T}, & \omega \in A_{5} .\end{cases}
$$

Then, for each fuzzy random variable, the expected value $E[G]$ by using the Aumann integral (11) is equal to

$$
E(G)=\int_{\Omega} G d P=(0.985,0.3525,0.3425)_{T} .
$$

Suppose the following data are a random sample from the population:

$$
\begin{aligned}
& \left\{(1.5,0.25,0.25)_{T},(-0.7,0.3,0.1)_{T},(0,0.5,0.5)_{T}\right. \\
& \quad(2,0.2,0.4)_{T},(0,0.5,0.5)_{T},(3,0.5,0.5)_{T},(0,0.5,0.5)_{T} \\
& \left.\quad(1.5,0.25,0.25)_{T},(3,0.5,0.5)_{T},(-0.7,0.3,0.1)_{T}\right\}
\end{aligned}
$$

Now, we generate 1,000 samples by using the bootstrap method. 


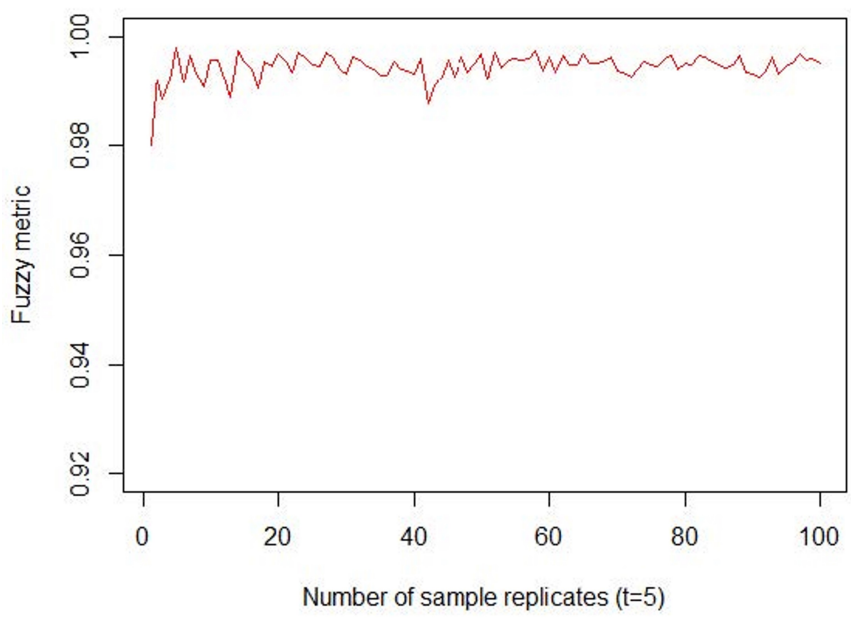

Figure 1. $M_{d_{1}}^{\prime}$ when $m(n) \rightarrow \infty$ and $t=5$.

In the next step, we calculate the mean of every 1,000 samples. To demonstrate better convergence, we compute the mean of the bootstrap method for the number of different iterations with 10 steps in the interval from 1 to 1,000. That is, we calculate the mean value in steps $10,20, \ldots, 1,000$ and display these on the chart. These values are shown in Figure 1

As shown in Figure 1 , when $m(n) \rightarrow \infty$ and $t=5$, the expectation of the random set tends to the sample mean by using the bootstrap method in the fuzzy metric, i.e.,

$$
M_{d_{1}}^{\prime}\left(\frac{1}{m(n)} \sum_{i=1}^{m(n)} Y_{n, i}, E\left(\operatorname{co} X_{1}\right), t\right) \rightarrow 1 \text { a.e. }
$$

In addition, the effect of value $t$ on the behavior of $M_{d_{1}}^{\prime}$ and the convergence rate can be seen in Figure 2. Using a low value of $t$ reduces the value of $M_{d_{1}}^{\prime}$ to 0 too quickly as the Pompeiu-Hausdorff distance increases. However, if a high value is used, the value of $M_{d_{1}}^{\prime}$ decreases too slowly. Consequently, the expert's opinion is important in determining the appropriate value of $t$.

\section{Conclusion}

When the uncertainty is fuzziness, as sometimes in the measurement of an ordinary length, the concept of a fuzzy metric space is more suitable. We have presented a new theorem for the study of the SLLN for fuzzy random variables in fuzzy metric spaces in the sense of George and Veeramani [10]. In addition, we have presented a definition of fuzzy metric spaces and fuzzy normed space for fuzzy sets and generalized the Radstrom embedding theorem, Lebesgue dominated convergence, and the Shapley-
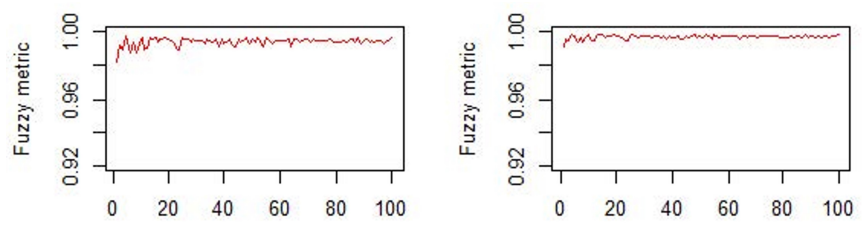

Number of sample replicates $(t=10)$

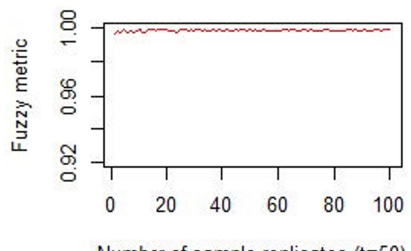

Number of sample replicates $(t=20)$

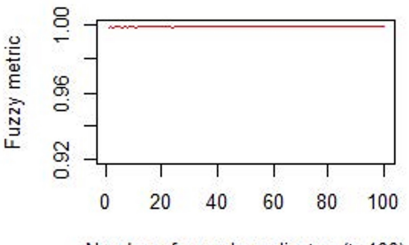

Number of sample replicates $(t=100)$

Figure 2. Behavior of $M_{d_{1}}^{\prime}$ when $m(n) \rightarrow \infty$ for different values of $t(=10,20,50$, and 100).

Folkman-Starr theorems, which are important tools to prove these theorems. Our main result is the first version of the SLLN, extending the result of Klement et al. [2] to the natural case of the fuzzy metric $M_{d_{1}}^{\prime}$. Given that the concept of distance and fuzzy sets is used in several fields such as data mining, the use of the fuzzy metric definition for fuzzy sets can initiate a new direction in research in these fields. As an application of the SLLN for fuzzy random variables in fuzzy metric space, we show that $\frac{1}{m(n)} \sum_{i=1}^{m(n)} Y_{n, i}$ tends to $E\left(\right.$ co $\left.X_{1}\right)$ in the fuzzy metric space for the bootstrap method. In fact, when an expert's opinion is important in determining distance, the value of $t$ in the fuzzy metric becomes significant.

\section{Conflict of Interest}

No potential conflict of interest relevant to this article was reported.

\section{References}

[1] M. L. Puri and D. A. Ralescu, "Fuzzy random variables," Journal of Mathematical Analysis and Applications, vol. 114, no. 2, pp. 409-422, 1986. https://doi.org/10.1016/ 0022-247X(86)90093-4

[2] E. Klement, M. L. Puri, and D. Ralescu, "Limit theorems for fuzzy random variables," Proceedings of the Royal Society of London. A. Mathematical and Physical Sciences, vol. 407, no. 1832, pp. 171-182. https: //doi.org/10.1098/rspa.1986.0091

[3] M. Lopez-Diaz and M. A. Gil, "Approximating integrably bounded fuzzy random variables in terms of the general- 
ized Hausdorff metric," Information Sciences, vol. 104, no. 3-4, pp. 279-291, 1998. https://doi.org/10.1016/S00200255(97)00068-6

[4] A. Colubi, M. Lopez-Diaz, J. S. Domiinguez-Menchero, and M. A. Gil, "A generalized strong law of large numbers," Probability Theory and Related Fields, vol. 114, no. 3, pp. 401-417, 1999. https://doi.org/10.1007/ s004400050229

[5] I. S. Molchanov, "On strong laws of large numbers for random upper semicontinuous functions," Journal of Mathematical Analysis and Applications, vol. 235, no. 1, pp. 349-355, 1999. https://doi.org/10.1006/jmaa.1999.6403

[6] S. Li and Y. Ogura, "Strong laws of large numbers for independent fuzzy set-valued random variables," Fuzzy Sets and Systems, vol. 157, no. 19, pp. 2569-2578, 2006. https://doi.org/10.1016/j.fss.2003.06.011

[7] Y. K. Kim, "Weak laws of large numbers for weighted sums of Banach space valued fuzzy random variables," International Journal of Fuzzy Logic and Intelligent Systems, vol. 13, no. 3, pp. 215-223, 2013. https://doi.org/10. 5391/IJFIS.2013.13.3.215

[8] I. Kramosil and J. Michalek, "Fuzzy metrics and statistical metric spaces," Kybernetika, vol. 11, no. 5, pp. 336-344, 1975.

[9] K. Menger, "Statistical metrics," Proceedings of the National Academy of Sciences of the United States of America, vol. 28, no. 12, pp. 535-537, 1942. https://doi.org/10. 1073/pnas.28.12.535

[10] A. George and P. Veeramani, "On some results in fuzzy metric spaces," Fuzzy Sets and Systems, vol. 64, no. 3, pp. 395-399, 1994. https://doi.org/10.1016/0165-0114(94) 90162-7

[11] S. Morillas, V. Gregori, G. Peris-Fajarnes, and P. Latorre, "A fast impulsive noise color image filter using fuzzy metrics," Real-Time Imaging, vol. 11, no. 5-6, pp. 417-428, 2005. https://doi.org/10.1016/j.rti.2005.06.007

[12] S. Morillas, V. Gregori, and A. Hervas, "Fuzzy peer groups for reducing mixed gaussian-impulse noise from color images," IEEE Transactions on Image Processing, vol. 18, no. 7, pp. 1452-1466, 2009. https://doi.org/10. 1109/TIP.2009.2019305
[13] S. Morillas, L. Gomez-Robledo, R. Huertas, and M. Melgosa, "Fuzzy analysis for detection of inconsistent data in experimental datasets employed in the development of the ciede2000 colour-difference formula," Journal of Modern Optics, vol. 56, no. 13, pp. 1447-1456, 2009. https://doi.org/10.1080/09500340902944038

[14] V. Gregori, J. J. Minana, and S. Morillas, "Some questions in fuzzy metric spaces," Fuzzy Sets and Systems, vol. 204, pp. 71-85, 2012. https://doi.org/10.1016/j.fss.2011.12.008

[15] B. Efron, "Bootstrap methods: another look at the jackknife," in Breakthroughs in Statistics. New York, NY: Springer, 1992, pp. 569-593. https://doi.org/10.1007/9781-4612-4380-9_41

[16] K. B. Athreya, "Strong law for the bootstrap," Statistics \& Probability Letters, vol. 1, no. 3, pp. 147-150, 1983. https://doi.org/10.1016/0167-7152(83)90063-9

[17] K. Athreya, M. Ghosh, L. Low, and P. Sen, "Laws of large numbers for bootstrappedU-statistics," Journal of Statistical Planning and Inference, vol. 9, no. 2, pp. 185194, 1984.https://doi.org/10.1016/0378-3758(84)90019-3

[18] B. Schweizer and A. Sklar, "Statistical metric spaces," Pacific Journal of Mathematics, vol. 10, no. 1, pp. 313334, 1960. https://doi.org/10.2140/pjm.1960.10.313

[19] E. P. Klement, R. Mesiar, and E. Pap, Triangular Norms. Dordrecht, The Netherlands: Kluwer Academic Publishers, 2000.

[20] L. Zadeh, "Fuzzy sets," Information and Control, vol. 8, no. 3, pp. 338-353, 1965. https://doi.org/10.1016/S00199958(65)90241-X

[21] M. L. Puri and D. A. Ralescu, "Strong law of large numbers for Banach space valued random sets," The Annals of Probability, vol. 11, no. 1, pp. 222-224, 1983.

[22] M. Hukuhara, "Integration des applications mesurables dont la valeur est un compact convexe," Funkcialaj Ekvacioj, vol. 10, no. 3, pp. 205-223, 1967.

[23] L. Stefanini, "A generalization of Hukuhara difference and division for interval and fuzzy arithmetic," Fuzzy Sets and Systems, vol. 161, no. 11, pp. 1564-1584, 2010. https://doi.org/10.1016/j.fss.2009.06.009 
[24] P. Diamond and P. E. Kloeden, Metric Spaces of Fuzzy Sets: Theory and Applications. Singapore: World Scientific Publishing, 1994.

[25] V. Lakshmikantham and R. N. Mohapatra, Theory of Fuzzy Differential Equations and Inclusions. London, UK: Taylor \& Francis, 2003.

[26] Z. Deng, "Fuzzy pseudo-metric spaces," Journal of Mathematical Analysis and Applications, vol. 86, no. 1, pp. 7495, 1982. https://doi.org/10.1016/0022-247X(82)90255-4

[27] O. Kaleva and S. Seikkala, "On fuzzy metric spaces," Fuzzy Sets and Systems, vol. 12, no. 3, pp. 215-229, 1984. https://doi.org/10.1016/0165-0114(84)90069-1

[28] M. A. Erceg, "Metric spaces in fuzzy set theory," Journal of Mathematical Analysis and Applications, vol. 69, no. 1, 205-230, 1979. https://doi.org/10.1016/0022-247X(79) 90189-6

[29] R. Saadati and S. Vaezpour, "Some results on fuzzy Banach spaces," Journal of Applied Mathematics and Computing, vol. 17, no. 1-2, pp. 475-484, 2005. https: //doi.org/10.1007/BF02936069

[30] S. Morillas, V. Gregori, G. Peris-Fajarnes, and A. Sapena, "New adaptive vector filter using fuzzy metrics," Journal of Electronic Imaging, vol. 16, no. 3, article no. 033007, 2007. https://doi.org/10.1117/1.2767335

[31] H. Radstrom, "An embedding theorem for spaces of convex sets," Proceedings of the American Mathematical Society, vol. 3, no. 1, pp. 165-169, 1952. https: //doi.org/10.2307/2032477

[32] I. Molchanov, Theory of Random Sets. London, UK: Springer, 2005. https://doi.org/10.1007/978-1-4471-73496

[33] R. J. Aumann, "Integrals of set-valued functions," Journal of Mathematical Analysis and Applications, vol. 12, no. 1, pp. 1-12, 1965. https://doi.org/10.1016/0022-247x(65) 90049-1

[34] G. Debreu, "Integration of correspondences," in Proceedings of the 5th Berkeley Symposium on Mathematical Statistics and Probability, Berkeley, CA, 1967.
[35] C. L. Byrne, "Remarks on the set-valued integrals of Debreu and Aumann," Journal of Mathematical Analysis and Applications, vol. 62, no. 2, pp. 243-246, 1978. https://doi.org/10.1016/0022-247X(78)90123-3

[36] K. Arrow and F. Hahn, General Competitive Analysis. San Francisco, CA: Holden-Day, 1971.

[37] M. L. Puri and D. A. Ralescu, "Limit theorems for random compact sets in Banach space," Mathematical Proceedings of the Cambridge Philosophical Society, vol. 97, no. 1, pp. 151-158, 1985. https://doi.org/10.1017/ S0305004100062691

[38] S. Csorgo, "On the law of large numbers for the bootstrap mean," Statistics \& Probability Letters, vol. 14, no. 1, pp. 1-7, 1992. https://doi.org/10.1016/0167-7152(92)90203-h

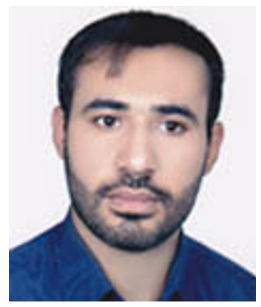

Reza Ghasemi is a Ph.D. student in the Department of Mathematics, Shahrood University of Technology, Iran. His research interests are probability theory, fuzzy theory, limit theorems, and image processing. E-mail: rezaghng@gmail.com.

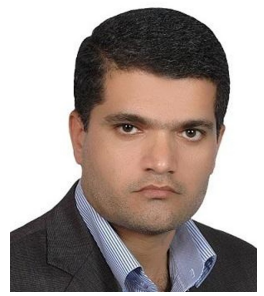

Mohammad Reza Rabiei is an Assistant Professor in the Department of Mathematics, Shahrood University of Technology, Iran. He earned his Ph.D. from Ferdowsi University of Mashhad. His research interests include statistical inference, fuzzy theory, interval-valued fuzzy, and regression analysis.

E-mail: rabiei1354@yahoo.com.

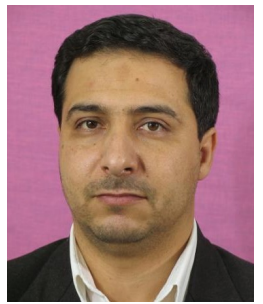

Ahmad Nezakati is an Associate Professor in the Department of Mathematics, Shahrood University of Technology, Iran. He earned his Ph.D. from Ferdowsi University of Mashhad. His research interests are probability theory, random polynomials, limit theorems, dependent random variables, and moment bounds.

E-mail: nezakati@shahroodut.ac.ir. 